\title{
A síndrome de Stevens-Johnson em pacientes neurocirúrgicos
}

\author{
Rui D’Avila', Eberval Gadelha Figueiredo², Manoel Jacobsen Teixeira ${ }^{3}$ \\ Divisão de Neurocirurgia do Hospital das Clínicas da Faculdade de Medicina da Universidade de São Paulo, \\ São Paulo, SP, Brasil.
}

\section{RESUMO}

Objetivo: Realizar uma revisão sobre os riscos aos quais pacientes neurocirúrgicos estão submetidos em relação à síndrome de Stevens-Johnson (SSJ). Métodos: Utilizaram-se as bases de dados Cochrane, Medline, Bireme/Lilacs, buscando-se artigos dos últimos seis anos que abordassem situações clínicas relacionadas à SSJ em pacientes neurocirúrgicos. Os trabalhos foram avaliados quanto a resultados, relevância clínica, desenho do estudo e nível de evidência. Resultados: A carbamazepina é comumente implicada, sendo o HLA-B*1502 fator de risco para tal afecção na população chinesa Han e associado à mortalidade. Outras populações foram testadas para tal HLA, não se encontrando resultados homogêneos. Ácido valproico, lamotrigina, fenitoína, fenobarbital e oxcarbazepina também estão relacionados. O EMPACT (Erythema Multiforme associated with Phenytoin And Cranial radiation Therapy) é observado em pacientes portadores de malignidades intracranianas submetidos à radiação, que iniciam fenitoína para prevenção ou tratamento de crises convulsivas. Em até $73 \%$ dos casos, esse eritema pode evoluir para SSJ. Conclusão: Pacientes neurocirúrgicos são frequentemente expostos a fatores causais de SSJ. Seu manejo consiste em suporte e suspensão da terapia envolvida. Procedimentos terapêuticos devem ser utilizados com cautela, levando-se em consideração sua real necessidade, HLA suscetíveis, idade e comorbidades.

\section{PALAVRAS-CHAVE}

Síndrome de Stevens-Johnson, neurocirurgia, anticonvulsivantes, antígenos HLA.

\section{ABSTRACT}

The Stevens-Johnson syndrome in neurosurgical patients

Objective: To review the risks to which neurosurgical patients are exposed in relation to Stevens-Johnson syndrome (SJS). Methods: We used the databases Cochrane, Medline and Bireme/Lilacs, seeking articles from the last six years about medical conditions related to SJS in neurosurgical patients. The papers were evaluated for results, clinical relevance, study design and level of evidence. Results: Carbamazepine is commonly involved. HLA-B*1502 is a risk factor for this disease in Han Chinese population and associated with mortality. Other populations were tested for HLA and no homogeneous findings were achieved. Valproic acid, lamotrigine, phenytoin, phenobarbital and oxcarbazepine are also related. The EMPACT (Erythema Multiforme Associated with Phenytoin and Cranial radiation Therapy) is observed in patients with intracranial malignancies undergoing radiation, with the concomitant use of phenytoin for the prevention or treatment of seizures. In up to $73 \%$ of the cases this erythema may progress to SJS. Conclusion: Neurosurgical patients are often exposed to causal factors of SJS. Support therapy and discontinuation of the drug involved are the management of this affection. Therapeutic procedures should be used with caution, taking into account their real needs, HLA susceptible, age and comorbidities.

\section{KEYWORDS}

Stevens-Johnson syndrome, neurosurgery, anticonvulsants, HLA antigens.

1 Estudante de Medicina da Universidade Federal do Rio Grande do Sul (UFRGS), Porto Alegre, RS, Brasil.

2 Neurocirurgião da Divisão de Neurocirurgia do Hospital das Clínicas da Universidade de São Paulo (USP), São Paulo, SP, Brasil.

3 Neurocirurgião, professor titular da Divisão de Neurocirurgia do Hospital das Clínicas da USP, São Paulo, SP, Brasil. 


\section{Introdução}

Reações cutâneas adversas a drogas desenvolvem-se com frequência, afetando $2 \%-3 \%$ de todos os pacientes hospitalizados. ${ }^{1}$ A síndrome de Stevens-Johnson (SSJ) caracteriza-se por uma reação idiossincrática grave, mais comumente desencadeada após 4 a 28 dias $^{2}$ de uso de medicações, em 30\%-50\% dos casos,${ }^{3-6}$ contudo, em crianças, o papel de infecções é mais acentuado. ${ }^{7,8}$

Manifesta-se tipicamente por lesões mucocutâneas de padrão eritematoso ou purpúrico, geralmente em forma de placas ou máculas, com formato de alvo, confluentes em tronco e face, atingindo pico máximo em quatro dias, ${ }^{3,9-11}$ sendo rotineiramente precedidas por mal-estar e febre, ${ }^{3,11}$ podendo levar à necrose do tecido acometido. ${ }^{3}$ As lesões em alvo são tipicamente descritas contendo três anéis: um central, brilhante e rosado, um mais pálido circundando o primeiro e um mais escuro que delimita exteriormente a lesão. ${ }^{10}$ Leucopenia significativa e sinal de Nikolski são outros achados que podem acompanhar a doença. ${ }^{12,13}$

Usualmente, membranas mucosas são lesadas (lábios, cavidade oral, conjuntiva, cavidade nasal, uretra, vagina, trato gastrointestinal e trato respiratório $)^{9} \mathrm{e}$, conceitualmente, a porcentagem de superfície corpórea envolvida deve ser igual ou inferior a $10 \% .{ }^{3}$ Podem haver complicações sistêmicas geralmente em pulmões, trato gastrointestinal, rins, sistema nervoso central, unhas, cabelos e outros. ${ }^{14}$

A incidência estimada varia de $1,1-7,1^{15,16} \mathrm{ca}$ sos/100.0000 pessoas/ano. A mortalidade em adultos se encontra entre $1 \%-3 \% ;{ }^{17} \mathrm{em}$ crianças, esse risco sobe para $7,5 \% .{ }^{18}$ Postula-se que na primavera a incidência possa ser mais elevada. ${ }^{19} \mathrm{O}$ sexo feminino é mais acometido $^{15}$ e não há uma idade mais afetada. ${ }^{15,20,21}$ As medicações comumente implicadas são: alopurinol, sulfonamidas, cefalosporinas, penicilinas, carbamazepina, lamotrigina, fenobarbital, piroxicam, nevirapina, entre outras. . $^{2,22-25}$

A patogênese não é precisamente conhecida; postula-se que seja relacionada a predisponentes metabólicos, ${ }^{26,27}$ major histocompatibility complex - I (MHC-I), ${ }^{28}$ linfócitos T CD $8+{ }^{29}$, Fas e L-faz, ${ }^{30}$ entre outros. Há indícios de que o metabolismo via citocromo P-450 possa estar comprometido nas reações a anticonvulsivantes aromáticos. Metabólitos que deveriam ser excretados pelas epóxido-hidrolases acabam não sendo eliminados. Estes, por sua vez, atuam como haptenos e iniciam uma resposta imunológica, causando necrose celular direta ou indireta, culminando em apoptose. ${ }^{31}$

Alguns fatores de risco implicados nessa doença são infecção por HIV (human immunodeficiency virus), fatores genéticos e infecções virais. Certos tipos de HLA (human leukocyte antigen), ${ }^{23}$ radioterapia, lúpus eritematoso sistêmico (LES), entre outros, também estão envolvidos. ${ }^{32}$

Dados acurados acerca da síndrome, tais como sua real incidência, são difíceis de serem obtidos em virtude do diagnóstico subjetivo (por vezes inconsistente) e da dificuldade em elencar os pacientes que estão de fato em risco para cada medicação. ${ }^{33,34}$

Objetivamos realizar uma revisão sobre os riscos de desenvolver SSJ aos quais pacientes neurocirúrgicos estão submetidos, descrevendo os principais fármacos e procedimentos terapêuticos envolvidos.

\section{Materiais e métodos}

A busca foi realizada utilizando-se as bases de dados Cochrane, Medline, Bireme/Lilacs, buscando-se artigos dos últimos seis anos a respeito de fármacos e situações clínicas relacionadas ao desenvolvimento de SSJ em pacientes neurocirúrgicos. Os trabalhos foram avaliados quanto a resultados, relevância clínica, desenho do estudo e nível de evidência.

\section{Resultados e discussão}

O paciente neurocirúrgico é exposto frequentemente a fatores causais de SSJ, sendo alvo de polifármacos e por vezes submetido à radiação. Mais recentemente, em estudo de sete anos de seguimento, Devi et al..$^{35}$ concluíram que anticonvulsivantes são a causa mais implicada com SSJ, especialmente no início do tratamento. ${ }^{34-36}$

O risco de usuários novos por 10.000 pessoas de desenvolver síndrome de Stevens Johnson (SSJ)/necrólise epidérmica tóxica (NET) é de aproximadamente 1,4 para carbamazepina, 2,5 para lamotrigina, 8,1 para fenobarbital, 8,3 para fenitoína e 0,4 para ácido valproico. ${ }^{36}$ A droga principal é a carbamazepina (mais de $80 \%$ dos casos). ${ }^{35}$ De acordo com estudos, as drogas que possivelmente são mais implicadas na gênese dessa afecção são carbamazepina, fenitoína e alopurinol, ${ }^{37}$ apresentando no estudo de Lin et al. ${ }^{37}$ risco relativo de 33,0 (IC 95\%, 4,3-255,6), 9,6 (IC 95\%, 2,0-46,6), 18 (IC 95\%, 2,2-149,5) e risco relativo com análise multivariada de 301,8 (IC 95\%, 13,6-6700,2), 290,8 (CI 95\%, 9,2-9239,3), 186,7 (IC $95 \%, 6,4-5458,2)$, respectivamente para esses fármacos. O intervalo de confiança de $95 \%$ apresenta-se bastante alargado, pela pequena quantidade de pacientes, visto que é uma afecção rara. Contudo, é importante que se observe que tal intervalo em nenhum dos casos ultrapassa a faixa de igualdade, situada no valor 1 , demonstrando significância quanto a esse aspecto. 
Com relação à carbamazepina, sua aplicação não se limita apenas ao controle de epilepsia, mas também ao controle de dor. ${ }^{1}$ De acordo com Czajkowski et al., ${ }^{1}$ a droga causa SSJ/NET em aproximadamente 14/100.000 usuários. Alguns estudos retrospectivos sugerem que ela seja o anticonvulsivante mais relacionado a SS, ${ }^{37}$ apresentando risco aumentado no início da instituição, ${ }^{36}$ manifestando-se geralmente após 15 dias de administração. ${ }^{2}$ É aventado que o HLA-B ${ }^{\star} 1502$ possa estar implicado na etiologia de tal afecção na população chinesa Han e mais associado à mortalidade, podendo ser rastreado antes do uso de carbamazepina. ${ }^{23}$ Além do rastreamento de HLA, testes com patches cutâneos poderiam ser utilizados para avaliar a predisposição a desenvolver a síndrome. ${ }^{23,38}$

Tal associação com HLA-B ${ }^{\star} 1502$ foi feita em diversos estudos, dentre os quais se destaca o realizado por Hung et al. ${ }^{23}$ com 91 casos e 144 controles, no qual se encontrou um odds ratio (OD) de 1.357 ( IC 95\%, 193,4-8838,3) para tal associação, podendo esse alelo ser fator crucial na patogênese dessa afecção por essa droga. Aventa-se que o HLA-B ${ }^{\star} 1502$ atuaria na apresentação antigênica e reconhecimento por células T. Outras hipóteses como deficiência na enzima epóxido-hidrolase 1 não foram confirmadas. ${ }^{39,40}$

Em estudo conduzido por Alfirevic et al. ${ }^{41}$ foi analisada uma população de caucasianos composta por 99 pacientes, levando-se em consideração também reações de hipersensibilidade à carbamazepina, além de SSJ. Vinte pacientes apresentaram alterações cutâneas, sendo 18 de hipersensibilidade, 1 de SSJ e 1 de NET. Nesse estudo não se encontrou relação entre hipersensibilidade à carbamazepina e o HLA- $B^{\star} 1502$, não sendo esse um marcador fidedigno de hipersensibilidade em população que não a chinesa Han, até o momento. Houve, porém, uma sugestão de que $\mathrm{HLA}^{*} 0702$ possa conferir certa proteção à reação a carbamazepina.

Com relação a outras populações, a testagem de HLA mostrou-se variada. Na população coreana, o HLA-B ${ }^{\star} 1502$ não se mostrou bom preditor de reação cutânea adversa à carbamazepina, entretanto o HLA$-B^{\star} 1512$ foi associado a SSJ ( $\mathrm{p}=0,011$ e OR 18$) .{ }^{42} \mathrm{Com}$ relação à população europeia, o HLA-A*3101 demonstrou associação, apresentando OR de 25,93 (CI 95\%, 4,93-116,18) ${ }^{43}$ Na população de Taiwan, foi realizado um estudo com 4.877 pacientes, no qual se avaliou a presença de HLA- $B^{\star} 1502$. Os indivíduos com a presença de tal HLA foram desaconselhados a utilizar carbamazepina, e para os outros iniciou-se tal medicação. Em nenhum dos pacientes com HLA-B ${ }^{\star} 1502$-negativo desenvolveu-se a síndrome, resultado muito abaixo do previsto, que seria de aproximadamente 10 casos, apresentando um valor $\mathrm{p}<0,001 .{ }^{44} \mathrm{~A}$ população malaia possui múltiplas etnias, e, em um estudo conduzido nessa população, o HLA-B ${ }^{\star} 1502$ também apresentou associação com SSJ induzida por carbamazepina. ${ }^{45} \mathrm{Na}$ população japonesa, há sugestão de associação com o HLA-B ${ }^{\star} 1511$ e o $B^{\star} 3101 .{ }^{46,47} \mathrm{Na}$ população tailandesa e indiana, o HLA- $B^{\star} 1502$ também foi associado. ${ }^{48,49}$

Portanto, é importante que se considerem os riscos pelos quais o paciente passará ao se introduzir essa medicação, avaliando-se sua real necessidade. Além disso, deve-se sempre lembrar dos efeitos adversos da carbamazepina, principalmente nos idosos..$^{50}$

Outros medicamentos utilizados em pacientes neurocirúrgicos também estão implicados na instalação de SSJ. A lamotrigina, um fármaco relativamente novo, vem sendo relacionada a SSJ, com o desenvolvimento da reação dentro das primeiras oito semanas de administração do medicamento, ${ }^{34,36}$ podendo ter eventual correlação com o HLA-B ${ }^{\star} 1502 .{ }^{51}$

O ácido valproico apresenta potencial baixo de causar SSJ quando utilizado isoladamente, levando em média mais de 365 dias para que essa reação ocorra. ${ }^{52}$ Contudo, acredita-se que possa aumentar a concentração sérica de outros antiepilépticos, colaborando para o aumento de reações adversas como rashs cutâneos. A associação de lamotrigina e ácido valproico causando SSJ já foi bem descrita. ${ }^{25}$

O uso de acetazolamida intravenosa também já foi associada a SSJ, principalmente em pacientes de ascendência indiana ou japonesa. ${ }^{53} \mathrm{~A}$ oxcarbazepina também já foi associada a SSJ, mantendo, assim como a carbamazepina, relação com HLA- ${ }^{\star}{ }^{\star} 15$, porém não com HLA-B ${ }^{\star} 1502$, descrito por Hung et al..$^{23}$ Isso se deve provavelmente ao fato de os dois fármacos possuírem estruturas bioquímicas semelhantes. ${ }^{54}$

Assim como para outros anticonvulsivantes, o risco de desenvolver SSJ com a administração simples de fenitoína é mais comum no início do tratamento, sendo, porém, muito raro (3\%-5\%); ${ }^{33}$ é improvável apenas com a aplicação de radioterapia isolada.$^{55-57}$ Porém, outra entidade descrita relacionada a SSJ é o EMPACT (Erythema Multiforme associated with Phenytoin And Cranial radiation Therapy). Tal entidade é observada principalmente em pacientes portadores de malignidades intracranianas que iniciam fenitoína para prevenção ou tratamento de crises convulsivas e são submetidos concomitantemente à radiação. Há o desenvolvimento de um eritema multiforme que em até $73 \%$ dos casos pode se converter em SSJ. Ahmed et al..$^{58}$ analisaram 24 pacientes com tal reação. Não foi evidenciada predisposição por idade ou sexo, o intervalo da administração de radiação e fármaco foi bastante variável e, em todos os casos, as lesões cutâneas se iniciaram no sítio de administração de radioterapia, sendo confundidas com dermatite aguda induzida por radiação. A extensão das lesões não mostrou relação com a dose de fenitoína ou 
de radiação utilizadas, e nenhum dos pacientes apresentou critérios para síndrome de hipersensividade induzida por dilantina. Sua etiopatogênese não é completamente entendida, porém hipóteses metabólicas e imunogênicas são as mais relacionadas. . $^{58,59}$

Há relatos também de desenvolvimento de reações eritematosas com uso de carbamazepina e radiação concomitantemente. ${ }^{60} \mathrm{O}$ uso de fenobarbital em conjunto com radiação também foi associado a SSJ. ${ }^{24} \mathrm{O}$ uso de corticoide e anticonvulsivante e a exposição à radiação associados também podem estar implicados no desenvolvimento dessa síndrome cutânea. . $9,61,62^{2}$

O tratamento da SSJ consiste basicamente no manejo correto das feridas, hidratação, suporte oftalmológico e nutricional. O uso de corticosteroides é controverso, podendo aumentar o risco de complicações, como infecções secundárias e sangramento gastrointestinal. ${ }^{63}$ Estudos não controlados mostram que plasmaferese ${ }^{64}$ e ciclosporina ${ }^{65,66}$ podem ser benéficas. Imunoglobulina intravenosa também pode ser outra opção terapêutica, ${ }^{67}$ e a retirada da droga implicada no processo também é de grande importância, ${ }^{13,31}$ assim como tratar infecções subjacentes. ${ }^{14}$ Idade avançada, comorbidades e extensão de pele envolvida estão relacionadas a pior prognóstico. $^{68}$

\section{Conclusão}

Os pacientes neurocirúrgicos estão constantemente expostos a procedimentos terapêuticos que podem desencadear a SSJ, e tal síndrome deve sempre ser lembrada quando o paciente está em uso de polifármacos e apresenta eritema. As drogas mais envolvidas são os anticonvulsivantes, principalmente no início de seu uso, sendo os principais: carbamazepina, fenitoína, fenobarbital, ácido valproico, lamotrigina, oxcarbazepina, entre outros. Alguns HLA estão mais associados ao desenvolvimento de SSJ e, se possível, devem ser rastreados em populações sabidamente com maior propensão. Pacientes chineses Han portadores do HLA-B ${ }^{\star} 1502$ possuem maior tendência de desenvolver SSJ se expostos a carbamazepina. Patches cutâneos também podem servir como testes para predição de SSJ em usuários de algumas drogas. A radiação possui importância, podendo desencadear SSJ principalmente se associada a anticonvulsivantes, podendo levar ao desenvolvimento de EMPACT.

Profissionais envolvidos nos cuidados a pacientes neurocirúrgicos devem sempre estar atentos para a SSJ, levando em consideração a real necessidade da utilização das principais drogas envolvidas no processo. Uma vez detectada, tal síndrome deve ser prontamente tratada, pois pode causar sequelas graves ou até mesmo a morte.

\section{Referências}

1. Czajkowski R, Weiss-Rostkowska V, Wankiewicz A, Drewa T, Placek W, Biedka M, et al. Stevens-Johnson syndrome induced by carbamazepine. Acta Pol Pharm. 2007;64(1):89-92.

2. Mockenhaupt M, Viboud C, Dunant A, Naldi L, Halevy S, Bouwes Bavinck JN, et al. Stevens-Johnson syndrome and toxic epidermal necrolysis: assessment of medication risks with emphasis on recently marketed drugs. The EuroSCARstudy. J Invest Dermatol. 2008;128(1):35-44.

3. Bastuji-Garin S, Rzany B, Stern RS, Shear NH, Naldi L, Roujeau JC. Clinical classification of cases of toxic epidermal necrolysis, Stevens-Johnson syndrome, and erythema multiforme. Arch Dermatol. 1993;129(1):92-6.

4. Roujeau JC. Stevens-Johnson syndrome and toxic epidermal necrolysis are severity variants of the same disease which differs from erythema multiforme. J Dermatol. 1997;24(11):726-9.

5. Roujeau JC. What is going on in erythema multiforme? Dermatology. 1994;188(4):249-50.

6. Assier H, Bastuji-Garin S, Revuz J, Roujeau JC. Erythema multiforme with mucous membrane involvement and Stevens-Johnson syndrome are clinically different disorders with distinct causes. Arch Dermatol. 1995;131(5):539-43.

7. Léauté-Labrèze C, Lamireau T, Chawki D, Maleville J, Taïeb A. Diagnosis, classification, and management of erythema multiforme and Stevens-Johnson syndrome. Arch Dis Child. 2000;83(4):347-52.

8. Wetter DA, Camilleri MJ. Clinical, etiologic, and histopathologic features of Stevens-Johnson syndrome during an 8-year period at Mayo Clinic. Mayo Clin Proc. 2010;85(2):131-8.

9. Hazin R, Ibrahimi OA, Hazin MI, Kimyai-Asadi A. StevensJohnson syndrome: pathogenesis, diagnosis, and management. Ann Med. 2008;40(2):129-38.

10. Letko E, Papaliodis DN, Papaliodis GN, Daoud YJ, Ahmed AR, Foster CS. Stevens-Johnson syndrome and toxic epidermal necrolysis: a review of the literature. Ann Allergy Asthma Immunol. 2005;94(4):419-36.

11. Stevens AM, Johnson FC. A new eruptive fever associated with stomatitis and ophthalmia: a report of two cases in children. Am J Dis Child. 1922;24:526-33.

12. Chave TA, Mortimer NJ, Sladden MJ, Hall AP, Hutchinson $\mathrm{PE}$. Toxic epidermal necrolysis: current evidence, practical management and future directions. $\mathrm{Br} \mathrm{J}$ Dermatol. 2005;153(2):241-53.

13. Knowles S, Shear NH. Clinical risk management of StevensJohnson syndrome/toxic epidermal necrolysis spectrum. Dermatol Ther. 2009;22(5):441-51.

14. Patel NN, Patel DN. Erythema multiforme syndrome. Am J Med. 2009;122(7):623-5.

15. Schöpf E, Stühmer A, Rzany B, Victor N, Zentgraf R, Kapp JF. Toxic epidermal necrolysis and Stevens-Johnson syndrome. An epidemiologic study from West Germany. Arch Dermatol. 1991;127(6):839-42.

16. Strom BL, Carson JL, Halpern AC, Schinnar R, Snyder ES, Shaw M, et al. A population-based study of StevensJohnson syndrome. Incidence and antecedent drug exposures. Arch Dermatol. 1991;127(6):831-8.

17. Carr DR, Houshmand E, Heffernan MP. Approach to the acute, generalized, blistering patient. Semin Cutan Med Surg. 2007;26(3):139-46.

18. Levi N, Bastuji-Garin S, Mockenhaupt M, Roujeau JC, Flahault A, Kelly JP, et al. Medications as risk factors of Stevens-Johnson syndrome and toxic epidermal necrolysis 
in children: a pooled analysis. Pediatrics. 2009;123(2):e297304.

19. Wanat KA, Anadkat MJ, Klekotka PA. Seasonal variation of Stevens-Johnson syndrome and toxic epidermal necrolysis associated with trimethoprim-sulfamethoxazole. J Am Acad Dermatol. 2009;60(4):589-94.

20. Power WJ, Ghoraishi M, Merayo-Lloves J, Neves RA, Foster CS. Analysis of the acute ophthalmic manifestations of the erythema multiforme/Stevens-Johnson syndrome/toxic epidermal necrolysis disease spectrum. Ophthalmology. 1995;102(11):1669-76.

21. Patterson R, Miller M, Kaplan M, Doan T, Brown J, Detjen $P$, et al. Effectiveness of early therapy with corticosteroids in Stevens-Johnson syndrome: experience with 41 cases and a hypothesis regarding pathogenesis. Ann Allergy. 1994;73(1):27-34.

22. Halevy S, Ghislain PD, Mockenhaupt M, Fagot JP, Bouwes Bavinck JN, Sidoroff A, et al. Allopurinol is the most common cause of Stevens-Johnson syndrome and toxic epidermal necrolysis in Europe and Israel. J Am Acad Dermatol. 2008;58(1):25-32.

23. Hung SI, Chung WH, Jee SH, Chen WC, Chang YT, Lee WR, et al. Genetic susceptibility to carbamazepine-induced cutaneous adverse drug reactions. Pharmacogenet Genomics. 2006;16(4):297-306.

24. Duncan KO, Tigelaar RE, Bolognia JL. Stevens-Johnson syndrome limited to multiple sites of radiation therapy in a patient receiving phenobarbital. J Am Acad Dermatol. 1999;40(3):493-6.

25. Yalçin B, Karaduman A. Stevens-Johnson syndrome associated with concomitant use of lamotrigine and valproic acid. J Am Acad Dermatol. 2000;43(5 Pt 2):898-9.

26. Shear NH, Spielberg SP. Anticonvulsant hypersensitivity syndrome. In vitro assessment of risk. J Clin Invest. 1988;82(6):1826-32.

27. Bachot N, Roujeau JC. Physiopathology and treatment of severe drug eruptions. Curr Opin Allergy Clin Immunol. 2001;1(4):293-8.

28. Nassif A, Bensussan A, Boumsell L, Deniaud A, Moslehi H, Wolkenstein $P$, et al. Toxic epidermal necrolysis: effector cells are drug-specific cytotoxic T cells. J Allergy Clin Immunol. 2004;114(5):1209-15.

29. Miyauchi H, Hosokawa H, Akaeda T, Iba H, Asada $Y$. T-cell subsets in drug-induced toxic epidermal necrolysis. Possible pathogenic mechanism induced by CD8-positive T cells. Arch Dermatol. 1991;127(6):851-5.

30. Viard I, Wehrli P, Bullani R, Schneider P, Holler N, Salomon $D$, et al. Inhibition of toxic epidermal necrolysis by blockade of CD95 with human intravenous immunoglobulin. Science. 1998;282(5388):490-3.

31. Knowles SR, Shear NH. Recognition and management of severe cutaneous drug reactions. Dermatol Clin. 2007;25(2):245-53.

32. Chan HL. Observations on drug-induced toxic epidermal necrolysis in Singapore. J Am Acad Dermatol. 1984;10(6):973-8.

33. Roujeau JC, Kelly JP, Naldi L, Rzany B, Stern RS, Anderson $\mathrm{T}$, et al. Medication use and the risk of Stevens-Johnson syndrome or toxic epidermal necrolysis. N Engl J Med. 1995;333(24):1600-7.

34. Rzany B, Correia O, Kelly JP, Naldi L, Auquier A, Stern R. Risk of Stevens-Johnson syndrome and toxic epidermal necrolysis during first weeks of antiepileptic therapy: a case-control study. Study Group of the International Case Control Study on Severe Cutaneous Adverse Reactions. Lancet. 1999;353(9171):2190-4.
35. Devi K, George S, Criton S, Suja V, Sridevi PK. Carbamazepine - the commonest cause of toxic epidermal necrolysis and Stevens-Johnson syndrome: a study of 7 years. Indian $\mathrm{J}$ Dermatol Venereol Leprol. 2005;71(5):325-8.

36. Mockenhaupt M, Messenheimer J, Tennis P, Schlingmann J. Risk of Stevens-Johnson syndrome and toxic epidermal necrolysis in new users of antiepileptics. Neurology. 2005;64(7):1134-8.

37. Lin MS, Dai YS, Pwu RF, Chen YH, Chang NC. Risk estimates for drugs suspected of being associated with Stevens-Johnson syndrome and toxic epidermal necrolysis: a case-control study. Intern Med J. 2005;35(3):188-90.

38. Sharma VK, Vatve M, Sawhney IM, Kumar B. Clinical spectrum of drug rashes due to antiepileptics. J Assoc Physicians India. 1998;46(7):595-7.

39. Gaedigk A, Spielberg SP, Grant DM. Characterization of the microsomal epoxide hydrolase gene in patients with anticonvulsant adverse drug reactions. Pharmacogenetics. 1994;4(3):142-53.

40. Green VJ, Pirmohamed M, Kitteringham NR, Gaedigk A, Grant DM, Boxer M, et al. Genetic analysis of microsomal epoxide hydrolase in patients with carbamazepine hypersensitivity. Biochem Pharmacol. 1995;50(9):1353-9.

41. Alfirevic A, Jorgensen AL, Williamson PR, Chadwick DW, Park BK, Pirmohamed M. HLA-B locus in Caucasian patients with carbamazepine hypersensitivity. Pharmacogenomics. 2006;7(6):813-8.

42. Kim SH, Lee KW, Song WJ, Kim SH, Jee YK, Lee SM, et al. Carbamazepine-induced severe cutaneous adverse reactions and HLA genotypes in Koreans. Epilepsy Res. 2011;97(1-2):190-7.

43. McCormack M, Alfirevic A, Bourgeois S, Farrell JJ, Kasperavičiūte D, Carrington M, et al. HLA-A*3101 and carbamazepine-induced hypersensitivity reactions in Europeans. N Engl J Med. 2011;364(12):1134-43.

44. Chen P, Lin JJ, Lu CS, Ong CT, Hsieh PF, Yang CC, et al. Carbamazepine-induced toxic effects and HLA-B*1502 screening in Taiwan. N Engl J Med. 2011;364(12):1126-33.

45. Chang CC, Too CL, Murad S, Hussein SH. Association of HLA-B ${ }^{\star} 1502$ allele with carbamazepine-induced toxic epidermal necrolysis and Stevens-Johnson syndrome in the multi-ethnic Malaysian population. Int $\mathrm{J}$ Dermatol. 2011;50(2):221-4.

46. Kaniwa N, Saito $\mathrm{Y}$, Aihara $\mathrm{M}$, Matsunaga $\mathrm{K}$, Tohkin $M$, Kurose $K$, et al. HLA- $B^{\star} 1511$ is a risk factor for carbamazepine-induced Stevens-Johnson syndrome and toxic epidermal necrolysis in Japanese patients. Epilepsia. 2010;51(12):2461-5.

47. Ozeki T, Mushiroda T, Yowang A, Takahashi A, Kubo M, Shirakata $Y$, et al. Genome-wide association study identifies HLA-A*3101 allele as a genetic risk factor for carbamazepineinduced cutaneous adverse drug reactions in Japanese population. Hum Mol Genet. 2011;20(5):1034-41.

48. Tassaneeyakul $\mathrm{W}$, Tiamkao S, Jantararoungtong $\mathrm{T}$, Chen $\mathrm{P}$, Lin SY, Chen WH, et al. Association between HLA-B ${ }^{\star} 1502$ and carbamazepine-induced severe cutaneous adverse drug reactions in a Thai population. Epilepsia. 2010;51(5):926-30.

49. Mehta TY, Prajapati LM, Mittal B, Joshi CG, Sheth JJ, Patel DB, et al. Association of HLA-B*1502 allele and carbamazepine-induced Stevens-Johnson syndrome among Indians. Indian J Dermatol Venereol Leprol. 2009;75(6):579-82.

50. Garcia JB, Ferro LS, Carvalho AB, Da Rocha RM, De Souza LM. Severe carbamazepine-induced cutaneous reaction in the treatment of post-herpetic neuralgia. Case report. Rev Bras Anestesiol. 2010;60(4):429-37. 
51. An DM, Wu XT, Hu FY, Yan B, Stefan H, Zhou D. Association study of lamotrigine-induced cutaneous adverse reactions and HLA-B*1502 in a Han Chinese population. Epilepsy Res. 2010;92(2-3):226-30.

52. Mockenhaupt M, Dunant A, Sidoroff A, Halevy S, Naldi L, Schlingmann J. Stevens-Johnson syndrome (SJS) and toxic epidermal necrolysis (TEN): the risk of antiepileptic drugs. In: Annual Meeting of the Society for Investigative Dermatology (SID). J Invest Dermatol. 2003;121:429.

53. Ogasawara K, Tomitsuka N, Kobayashi M, Komoribayashi $\mathrm{N}$, Fukuda T, Saitoh H, et al. Stevens-Johnson syndrome associated with intravenous acetazolamide administration for evaluation of cerebrovascular reactivity. Case report. Neurol Med Chir (Tokyo). 2006;46(3):161-3.

54. Lin LC, Lai PC, Yang SF, Yang RC. Oxcarbazepine-induced Stevens-Johnson syndrome: a case report. Kaohsiung J Med Sci. 2009;25(2):82-6.

55. Howell WR, Knight AL, Scruggs HJ. Stevens-Johnson syndrome after radiotherapy. South Med J. 1990;83(6):681-3.

56. Ridgway HB, Miech DJ. Erythema multiforme (StevensJohnson syndrome) following deep radiation therapy. Cutis. 1993;51(6):463-4.

57. Pandya AG, Kettler AH, Bruce S. Radiation-induced erythema multiforme. An unusual presentation with elastic tissue phagocytosis. Int J Dermatol. 1989;28(9):600-2.

58. Ahmed I, Reichenberg J, Lucas A, Shehan JM. Erythema multiforme associated with phenytoin and cranial radiation therapy: a report of three patients and review of the literature. Int J Dermatol. 2004;43(1):67-73

59. Cockey GH, Amann ST, Reents SB, Lynch JW Jr. StevensJohnson syndrome resulting from whole-brain radiation and phenytoin. Am J Clin Oncol. 1996;19(1):32-4.

60. Chevenet C, Ferrier MC, Reverte M, Tao Y, Perea R, Roger $\mathrm{H}$, et al. [Erythema multiforme caused by the combination of carbamazepine and cerebral irradiation]. Ann Dermatol Venereol. 1992;119(11):929-31.

61. Delattre JY, Safai B, Posner JB. Erythema multiforme and Stevens-Johnson syndrome in patients receiving cranial irradiation and phenytoin. Neurology. 1988;38(2):194-8.
62. Janinis J, Panagos G, Panousaki A, Skarlos D, Athanasiou $\mathrm{E}$, Karpasitis N, et al. Stevens-Johnson syndrome and epidermal necrolysis after administration of sodium phenytoin with cranial irradiation. Eur $\mathrm{J}$ Cancer. 1993;29A(3):478-9.

63. Arévalo JM, Lorente JA, González-Herrada C, JiménezReyes J. Treatment of toxic epidermal necrolysis with cyclosporin A. J Trauma. 2000;48(3):473-8.

64. Mittmann N, Chan B, Knowles S, Cosentino L, Shear N. Intravenous immunoglobulin use in patients with toxic epidermal necrolysis and Stevens-Johnson syndrome. Am J Clin Dermatol. 2006;7(6):359-68.

65. Lissia M, Figus A, Rubino C. Intravenous immunoglobulins and plasmapheresis combined treatment in patients with severe toxic epidermal necrolysis: preliminary report. $\mathrm{Br} \mathrm{J}$ Plast Surg. 2005;58(4):504-10.

66. Shortt R, Gomez M, Mittman N, Cartotto R. Intravenous immunoglobulin does not improve outcome in toxic epidermal necrolysis. J Burn Care Rehabil. 2004;25(3):246-55.

67. Kardaun SH, Jonkman MF. Dexamethasone pulse therapy for Stevens-Johnson syndrome/toxic epidermal necrolysis. Acta Derm Venereol. 2007;87(2):144-8.

68. Prins C, Kerdel FA, Padilla RS, Hunziker T, Chimenti S, Viard I, et al. Toxic epidermal necrolysis-intravenous immunoglobulin. Treatment of toxic epidermal necrolysis with high-dose intravenous immunoglobulins: multicenter retrospective analysis of 48 consecutive cases. Arch Dermatol. 2003;139(1):26-32.

\section{Endereço para correspondência}

Rui D’Ávila

Rua José Gomes, 123/407, Tristeza

91910-280 - Porto Alegre, RS, Brasil

Telefone: (51) 9889-5247

E-mail: rui_davila@hotmail.com 